\title{
Ga-68- and Cu-64-Labeled NOTA-Albumin Conjugates for PET Sentinel Lymph Node Imaging
}

\author{
Eik Schiller, ${ }^{1}$ Ralf Bergmann, ${ }^{2}$ Gerd Wunderlich, ${ }^{3}$ \\ Michael Andreeff, ${ }^{3}$ Anita Jacob, ${ }^{2}$ and Hans-Jürgen Pietzsch ${ }^{2}$ \\ ${ }^{1}$ ROTOP Pharmaka AG, Bautzner Landstraße 400, 01328 Dresden, Germany \\ ${ }^{2}$ Institute of Radiopharmaceutical Cancer Research, Helmholtz-Zentrum Dresden-Rossendorf, \\ P.O. Box 510119, 01314 Dresden, Germany \\ ${ }^{3}$ Department of Nuclear Medicine, University Hospital, "Carl Gustav Carus" Fetscherstraße 74, \\ 01307 Dresden, Germany
}

Correspondence should be addressed to Eik Schiller; eisc@rotop-pharmaka.de

Received 17 December 2012; Accepted 3 January 2013

Academic Editors: N. Gillings, P. Lass, and N. Motomura

Copyright (C) 2013 Eik Schiller et al. This is an open access article distributed under the Creative Commons Attribution License, which permits unrestricted use, distribution, and reproduction in any medium, provided the original work is properly cited.

Our objective was to develop and evaluate Ga-68- and $\mathrm{Cu}$-64-labeled albumin conjugates for PET imaging of sentinel lymph nodes. Four different albumin conjugates were prepared starting from NOTA-HSA. The lymph node uptake of Ga-68- and Cu-64-labeled albumin conjugates was investigated after subcutaneous injection into the foot pad of Wistar rats. A pig model was utilized for further biological evaluation of the lymph node uptake. For all the four conjugates, radiolabeling with Ga-68 and Cu-64 resulted in $>95 \%$ radiochemical yield. Denatured and mannosylated Ga-68 NOTA-HSA revealed the highest popliteal lymph node uptake in rats $(2.78 \% \pm 0.38 \%$ ID and $6.13 \% \pm 1.13 \%$ ID $10 \mathrm{~min}$ and $60 \mathrm{~min}$ p.i., resp.). The popliteal lymph node reached its maximum activity after approximately $120 \mathrm{~min}$ and remained constant for denatured and mannosylated Cu-64 NOTA-HSA at least up to 240 min p.i. In a pig model, $2 \%$ of the injected dose of this compound was found in the sentinel lymph node 60 min after subcutaneous injection. In conclusion, PET imaging of sentinel lymph nodes with Ga-68- and Cu-64-labeled denatured NOTA-Man-HSA could be successfully demonstrated and deserves further investigations.

\section{Introduction}

Sentinel lymph node (SLN) diagnosis is an important field in nuclear medicine, and its benefit has been proven in many cancer types, mainly breast cancer and melanoma. The sentinel lymph node is the first lymph node in the drainage basin of a primary tumor. SLN detection is a two-step procedure. In the first step, SLNs are imaged by means of planar lymphoscintigraphy. Recently, the advantage of SPECT/CT in cases with difficult anatomical location of sentinel nodes was reported as an alternative imaging modality $[1,2]$. In the second step of the procedure, SLNs are intraoperatively detected by a hand-held gamma camera, excised and pathologically examined. If the sentinel node is free of metastatic cells, the likelihood of general nodal metastasis is very low.
In general, technetium-99m-labeled colloids in combination with blue dyes are used to detect the sentinel lymph node. The labeled colloids are injected beneath the skin surrounding the primary tumor or alternatively directly into the tumor. After entering the lymphatic vessels, colloids use the same drainage pathway as potential metastatic cancer cells and are eventually retained in the first draining lymph node (sentinel node) by phagocytosis or mechanical trapping. In Europe, mainly the colloids based on denatured human serum albumin are used, whereas in USA; sulphur colloid is preferred.

There are two crucial factors in the performance of Tc$99 \mathrm{~m}$ colloids in SLN mapping. First, the agent has to be quickly transported from the site of injection to ensure the detection of SLNs in close proximity. Second, a high and persistent lymph node accumulation is necessary for 
the imaging and the intraoperative detection of the nodes. Both processes are mainly influenced by the size of the particles. As a compromise between fast transport of small particles from the site of injection and high lymph node uptake of larger colloids, nowadays a particle size of 10$200 \mathrm{~nm}$ is favoured [3-6].

A tailor-made Tc-99m radiopharmaceutical for SLN diagnosis based on dextran (Lymphoseek) is currently in clinical phase III studies $[7,8]$. In this case, $7 \mathrm{~nm}$-sized dextran as carrier molecule is additionally loaded with mannosyl units to enhance the receptor-mediated uptake of the radiopharmaceutical into the lymph nodes. Preliminary data indicated a similar lymph node uptake like filtered sulphur colloid, whereas injection site clearance is significantly faster [9]. The development of a positron-emitting agent would further improve the image resolution and the localization of SLNs by using PET/CT. Especially for SLNs in the abdomen, this is crucial for radiotherapy planning [10]. A recent paper by Heuveling et al. describes the preclinical results of $\mathrm{Zr}$ 89 nanocolloidal albumin PET/CT in head and neck cancer [11]. Choi and coworkers utilized the generator-derived Ga68 as PET nuclide and labeled mannosylated human serum albumin (MSA) for SLN detection in rodents [12].

Most of the currently available commercial technetium$99 \mathrm{~m}$ kits for sentinel lymph node scintigraphy contain colloids of denatured albumin. In our study, we investigated the effect of human serum albumin (HSA) denaturation in combination with mannosylation and compared the biological performance of this agent with that of the nondenatured HSA as well as probes without mannosyl units. We provide data on the biodistribution of Ga-68-labeled conjugates and the longterm biokinetic studies of one conjugate labeled with $\mathrm{Cu}-64$.

\section{Materials and Methods}

2.1. General. p-SCN-Bn-NOTA $\times 3 \mathrm{HCl}(\mathrm{NOTA}-\mathrm{NCS})$ was purchased from Macrocyclics. Tetra-O-acetyl mannosyl isothiocyanate was synthesized according to the procedure of Kühne et al. [13].

HSA solution (20\%) was purchased from C.A.F.-D.C.F., Belgium. Poloxamer 238 was purchased from BTC, Cologne, Germany. Gallium-68 was eluted from a Ge-68/Ga-68 generator (iThemba LABS, South Africa). Copper-64 was produced with a cyclotron CYCLONE $18 / 9$ via the ${ }^{64} \mathrm{Ni}(\mathrm{p}, \mathrm{n}){ }^{64} \mathrm{Cu}$ reaction according to the method of Thieme and co-workers [14].

TLC plates were scanned on a TLC analyzer (RITA, Raytest, Straubenhardt, Germany). For ultrafiltration, a Heraeus Labofuge 400R was used with a Jumbosep centrifugal device (Pall) with a molecular weight cut-off of $30 \mathrm{kDa}$. Samples were centrifuged at $2500 \mathrm{~min}^{-1}$ and $10^{\circ} \mathrm{C}$ for 60 minutes. Mass spectrometry was performed on an Autoflex MALDITOF/TOF (Bruker Daltonics) with dihydroxy benzoic acid (NOTA-HSA) and 3-hydroxypicolinic acid (NOTA-ManHSA) as matrices. Particle size distribution was determined with a Zetasizer Nano ZS (Malvern). PET data of rats was acquired using a microPET P4 (Siemens preclinical solutions, Knoxville, TN, USA) scanner. For the pig experiment, a
Sensation Biograph 16 Hi-REZ Siemens/CTI, Knoxville, TN, USA, was utilized.

2.2. Synthesis of NOTA-HSA. Two milliliters of a sodium tetraborate solution $(0.1 \mathrm{M}, \mathrm{pH} 9.2)$ was added to $3.4 \mathrm{mg}$ $(6.1 \mu \mathrm{mol}) \mathrm{p}-\mathrm{SCN}-\mathrm{Bn}-\mathrm{NOTA} \times 3 \mathrm{HCL}$. After complete dissolution of the solid in an ultrasonic bath, the solution was mixed with $100 \mu \mathrm{L}$ HSA solution $(20 \mathrm{mg} ; 0.3 \mu \mathrm{mol}$ ) in a $2 \mathrm{~mL}$ Eppendorf Protein LoBind Tube. The reaction mixture was treated in an Eppendorf Thermomixer at $30^{\circ} \mathrm{C}$ and $300 \mathrm{~min}^{-1}$ for $18 \mathrm{~h}$. Low molecular weight impurities were separated by ultrafiltration at $10^{\circ} \mathrm{C}$ (fivefold washing with water). The residual solution was filled into $10 \mathrm{~mL}$ glass vials and lyophilized. The number of conjugated NOTA per HSA was determined by MALDI-MS. The difference between the peak abundance of NOTA-HSA $(71,725 \mathrm{Da})$ and native HSA $(67,000)$ was divided by the MW of p-SCN-Bz-NOTA as trisodium salt due to the basic reaction mixture $(M=$ $626 \mathrm{~g} / \mathrm{mol})$. Eight NOTA chelators per HSA molecule were calculated.

2.3. Conjugation of Mannose to NOTA-HSA. Tetra-O-acetyl mannosyl isothiocyanate $(33 \mathrm{mg}$ ) and $30 \mathrm{mg}$ NOTA-HSA lyophilisate were dissolved in $30 \mathrm{~mL} 0.25 \mathrm{M}$ carbonate buffer $\mathrm{pH}$ 9.0. The reaction mixture was slowly stirred at room temperature for 18 hours. The solution was filtered with a syringe filter (pore size $0.45 \mu \mathrm{m}$ ). Unbound mannose was removed by ultrafiltration at $10^{\circ} \mathrm{C}$ (fivefold washing with water). The residual solution was filled into $10 \mathrm{~mL}$ glass vials and lyophilized. The number of conjugated mannosyl units was determined by MALDI-MS. The difference between the peak abundance of NOTA-Man-HSA $(75,585 \mathrm{Da})$ and NOTA-HSA $(71,725 \mathrm{Da})$ as a starting material was divided by the MW of mannosyl isothiocyanate $(M=221 \mathrm{~g} / \mathrm{mol})$. Seventeen sugar units per HSA molecule were calculated.

2.4. Denaturation of HSA Conjugates. In a volume of $20 \mathrm{~mL}$ water, $10 \mathrm{mg}$ of the HSA conjugate, $40 \mathrm{mg}$ of Poloxamer 238, and $11 \mathrm{mg}$ of $\mathrm{Na}_{2} \mathrm{HPO}_{4} \times 2 \mathrm{H}_{2} \mathrm{O}$ were subsequently dissolved. The $\mathrm{pH}$ was adjusted to 7.3 with $0.1 \mathrm{M} \mathrm{HCl}$, and the volume of the solution was adjusted with water to give a total of $40 \mathrm{~mL}$. The solution was then heated at $80^{\circ} \mathrm{C}$ for $30 \mathrm{~min}$. After cooling down to room temperature, the solution was filtered with a syringe filter (pore size $0.45 \mu \mathrm{m}$ ). Aliquots of $2 \mathrm{~mL}$ were filled into $10 \mathrm{~mL}$ glass vials, and the solvent was removed by lyophilization.

2.5. Ga-68 and Cu-64 Labeling of NOTA-HSA, NOTAHSA denat, NOTA-Man-HSA, and NOTA-Man-HSA denat One milliliter of ammonium acetate buffer (0.5 M, pH 5.0) was added to a $10 \mathrm{~mL}$ vial containing $0.5 \mathrm{mg}$ of the HSA conjugate. Subsequently, $0.3 \mathrm{~mL}$ of Ga-68 eluate (100$250 \mathrm{MBq}$ ) was added. After $15 \mathrm{~min}$ at room temperature, $0.25 \mathrm{~mL}$ of aqueous EDTA solution (0.04 M, pH 4) was added to the reaction mixture. Five minutes later, the radiochemical yield was determined by thin layer chromatography with chromatography paper MN128 (Macherey-Nagel) as a stationary phase and $0.1 \mathrm{M}$ citrate buffer $\mathrm{pH} 5.0$ as a mobile 
phase. Radiolabeled HSA conjugates remain at the starting point, whereas unbound radionuclide (as citrate or EDTA complex) and Ga-68 NOTA-NCS move with the solvent front $\left(R_{f}=0.9-1.0\right)$. The size of the radiolabeled conjugates was analyzed by the filtration of an aliquot through a filter with $80 \mathrm{~nm}$ pore size (Whatman). In an analogous manner, NOTA-Man-HSA $A_{\text {denat }}$ was labeled with $\mathrm{Cu}-64$ by adding $100 \mu \mathrm{L}$ of (ca. $100 \mathrm{MBq})\left[{ }^{64} \mathrm{Cu}\right] \mathrm{CuCl}_{2}$ solution $(0.1 \mathrm{~N} \mathrm{HCl})$. For in vivo experiments, the addition of EDTA was omitted, whereas $1 \mathrm{~mL}$ of HEPES (1M, pH 8) was added to the reaction mixture for $\mathrm{pH}$ adjustment.

2.6. In Vivo Studies. The animal research committee of the Regierungspräsidium Dresden approved the animal facilities and the experiments according to the institutional guidelines and the German animal welfare regulations. The experimental procedure used conforms to the European Convention for the Protection of Vertebrate Animals Used for Experimental and Other Scientific Purposes (ETS no. 123), to the Deutsches Tierschutzgesetz, and to the Guide for the Care and Use of Laboratory Animals published by the US National Institutes of Health (DHEW Publication no. (NIH) 82-23, Revised 1996, Office of Science and Health Reports, DRR/NIH, Bethesda, MD, USA). The Wistar rats (Wistar Unilever, HsdCpb: Wu, Harlan Winkelmann GmbH, Borchen, Germany, 100-150 g body weight) were housed under standard conditions with free access to standard food and tap water.

The biodistribution of Ga-68- and Cu-64-labeled HSA conjugates was studied after subcutaneous application into the foot pad or i.v. application.

The biodistribution of the radiolabeled compounds was studied in 4 male rats of each group at 10 and 60 min after tracer injection. The animals were anesthetized with desflurane (Suprane, Baxter Healthcare Corporation, Deerfield, IL, USA) $(7.0 \%-10.0 \% \mathrm{v} / \mathrm{v}$ in 30\% oxygen), and i.v. Ga-68 $108 \pm$ $20 \mathrm{MBq} / \mathrm{kg}$ BW (BW $135 \pm 28$ g), i.v. Cu-64 4.0 $\pm 0.4 \mathrm{MBq} / \mathrm{kg}$ BW (BW $118 \pm 3 \mathrm{~g}$ ), and intrafoot Ga-68 $55 \pm 8 \mathrm{MBq} / \mathrm{kg}$ (BW $130 \pm 21 \mathrm{~g}$ ), and intrafoot $\mathrm{Cu}-646.4 \pm 0.4 \mathrm{MBq} / \mathrm{kg}$ (BW $136 \pm 7 \mathrm{~g})$ aliquots were administered i.v. into a tail vein in $500 \mu \mathrm{L}$ and subcutaneously into the foot in $100 \mu \mathrm{L}$ isotonic $\mathrm{NaCl}$ or electrolyte solution E153 (Serumwerk Bernburg AG, Bernburg, Germany). After the recovery from anesthesia, rats were again anaesthetized at 10 or $60 \mathrm{~min}$ after tracer injection, respectively. The blood was withdrawn by heart puncture, and the animals were euthanized. Organs and tissues were removed, dried, and weighed, and the radioactivity was measured in a cross-calibrated well counter (WIZARD, Automatic Gamma Counter, Perkin Elmer, Waltham, MA, USA) or dose calibrator (Aktivimeter Isomed 2000; MEDNuklear-Medizintechnik, Dresden, Germany). The data were decay corrected and normalized to the amount of injected activity calculated from the activity of injection syringes before and after injection and expressed as the percentage of injected activity (\%ID) or the standardized uptake values in biodistribution studies (SUVbio, SUVbio = (tissue activity/tissue weight)/(total given activity/rat body weight)). Values are quoted as mean \pm standard deviation (mean \pm SD) for a group of animals.
2.7. PET Studies on Rats and Pig. Positron emission tomography experiments were performed on male Wistar rats. The animals were anesthetized through inhalation of desflurane (7\%-10\% Suprane) in 30\% oxygen/air (gas flow, $1 \mathrm{~L} / \mathrm{min}$ ). Rats were positioned and immobilized prone with their medial axis parallel to the axial axis of the scanner and their thorax, abdomen, and hind legs near to the center of the field of view of the micro-PET P4 (Siemens preclinical solutions, Knoxville, TN, USA) scanner. For attenuation correction, a 10 min transmission scan was obtained using a rotating Co57 point source before the tracer injection and collection of the emission scans.

The activity of the injection solution $(0.1 \mathrm{~mL})$ in a $1 \mathrm{~mL}$ hypodermic syringe was measured in the well counter cross-calibrated with the PET-scanner. The emission scan of 240 min PET acquisition was started, and with a delay of $30 \mathrm{~s}$, the injection of the radiotracer subcutaneously into the left foot was initiated, and the applied activities were for Ga-68: $29.5 \pm 3.2 \mathrm{MBq}(n=2)$ and $\mathrm{Cu}-64: 3.8 \pm$ $0.5 \mathrm{MBq}(n=4)$. Data acquisition was performed in $3 \mathrm{D}$ list mode. Emission data were collected continuously. The list mode data were sorted into sinograms with 50 frames $(15 \times 10 \mathrm{~s}, 5 \times 30 \mathrm{~s}, 5 \times 60 \mathrm{~s}, 4 \times 300 \mathrm{~s}$, and, $21 \times 600 \mathrm{~s})$. The data were decay, scatter, and attenuation corrected. The frames were reconstructed by an ordered subset expectation maximization applied to 3D (OSEM3D) sinograms with 14 subsets, 15 OSEM3D iterations, 25 maximum a posteriori (MAP) iterations, and $1.8 \mathrm{~mm}$ resolution using the FastMAP algorithm. The pixel size was 0.07 by $0.12 \mathrm{~cm}$, and the resolution in the center of field of view was $1.8 \mathrm{~mm}$. No correction for partial volume effects was applied. The image volume data were converted to Siemens ECAT7 format for further processing. The image files were then processed using the ROVER software (ABX GmbH, Radeberg, Germany). Masks for defining the three-dimensional regions of interest (ROI) were set, and the ROI's were defined by thresholding, and ROI time-activity curves (TACs) were for the subsequent data analysis. The ROI data and TAC were further analyzed using $R$ ( $R$ is available as Free Software under the terms of the Free Software Foundation's GNU General Public License in source code form) and especially developed program packages (Jörg van den Hoff, Helmholtz-Zentrum DresdenRossendorf, Dresden, Germany). The standardized uptake values $($ SUVPET, SUVPET $=($ activity $/ \mathrm{mL}$ tissue $) /($ injected activity/body weight, $\mathrm{mL} / \mathrm{g}$ )) were calculated in ROI.

The pig experiment was performed according to a previously published procedure by Tafra et al. [15]. Briefly, an anesthetized pig was administered intradermally with $45 \mathrm{MBq}$ Ga-68 NOTA-Man-HSA denat $_{\text {in }} 0.5 \mathrm{~mL}$. The injection site was placed just distal to the hind and forelimb joints of each hind leg. Injection was made at four points equidistant around the mock site in each extremity. PET/CT scans were acquired $15 \mathrm{~min}$ and $60 \mathrm{~min}$ after injection.

\section{Results and Discussion}

3.1. Synthesis of NOTA-HSA, NOTA-HSA denat, NOTA-Man$H S A$, and NOTA-Man-HSA denat. All conjugates were prepared starting from NOTA-HSA which was synthesized by 


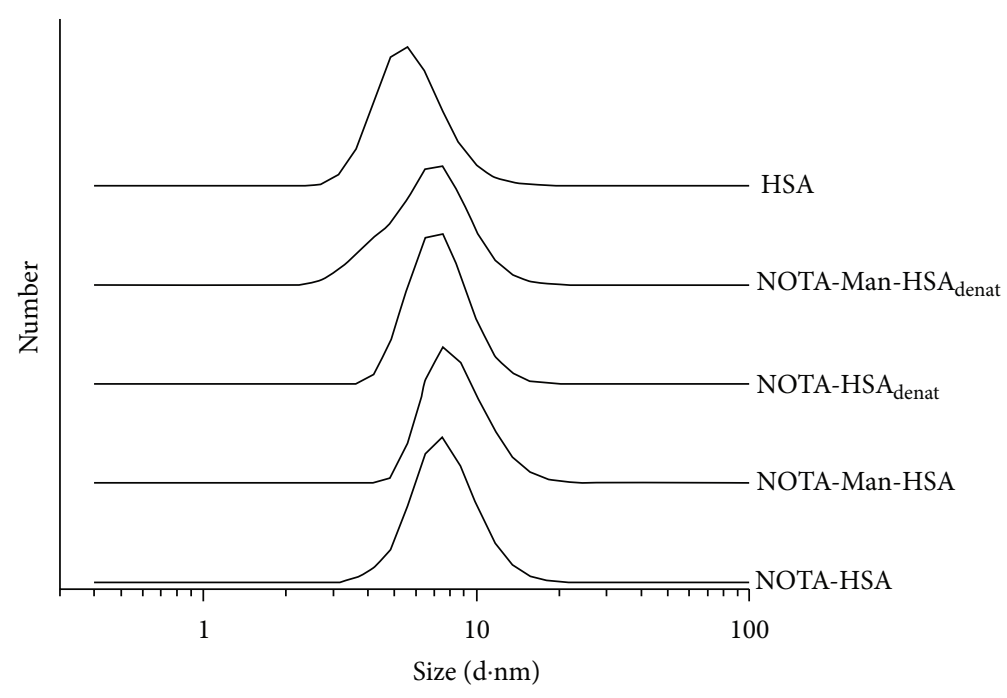

FIGURE 1: Size distribution of albumin conjugates and native HSA.

conjugation of an isothiocyanate derivative of NOTA to $\varepsilon$-amino groups of HSA lysine side chains. The number of conjugated chelators was determined by MALDI-MS, comparing between the mean molecular weights of NOTAHSA and native HSA and dividing the difference by the MW of NOTA-NCS. By using a 10-fold or 20-fold molar excess of NOTA, approximately 4 and 8 chelators could be conjugated per albumin, respectively. In subsequent studies, it turned out that using albumin with 4 NOTA units results in low radiochemical yield (ca. $87 \%$ ) of the corresponding denatured conjugates. Therefore, further studies were conducted by using albumin with 8 conjugated chelators.

In the second step, in a similar manner, tetra-O-acetyl mannosyl isothiocyanate was bound to NOTA-HSA. A 200fold molar excess of mannose was used to fix as much mannose as possible to all sterically available $\varepsilon$-amino groups of lysine side chains. The $\mathrm{O}$-acetyl groups of the sugar are cleaved under the used reaction conditions $(0.25 \mathrm{M}$ carbonate buffer $\mathrm{pH}$ 9). This was proven by preliminary experiments with penta-O-acetyl mannose treated under identical conditions. ${ }^{1} \mathrm{H}$ NMR of the reaction mixture of that compound was performed to show that no more proton signals of mannose$\mathrm{O}$-acetyl $\mathrm{CH}_{3}$ groups were found. Instead, a singlet for acetate $\mathrm{CH}_{3}$ was detected. For mannosylated NOTA-HAS, the number of attached mannose units was again calculated from the results of MALDI-MS. An average of 17 sugars per NOTAHSA was calculated. In the work of Choi and co-workers, 7 NOTA chelators and 11 mannose units were conjugated to HSA [12]. Therefore, the number of NOTA molecules was comparable to our conjugates; the higher number of mannose units can be explained by the larger excess of sugar in our case. Moreover, we used a mannose derivative where the isothiocyanate group is directly attached to the 1-position of the sugar ring. This compound is presumably much more soluble in water than the compound used by Choi et al. containing an aromatic ring in its structure.
Both conjugates, NOTA-HSA and NOTA-Man-HSA, were subsequently subjected to heat denaturation. The presence of the surfactant Poloxamer 238 prevents the colloids from extensive conglomeration and precipitation. The formulation was chosen according to Nanocoll (GE Healthcare), the most widely used Tc-99m radiopharmaceutical for sentinel lymph node mapping in Europe.

Conjugates were furthermore characterized by size distribution analysis (Figure 1). The average size of NOTAHSA, NOTA-Man-HSA, NOTA-HSA denat , and NOTA-Man$\mathrm{HSA}_{\text {denat }}$ was $7.8 \pm 2.0,8.3 \pm 2.2,7.0 \pm 1.8$, and $8.1 \pm 1.9 \mathrm{~nm}$, respectively. The differences in average size are very small and will not have any impact on the biodistribution of the agents. For comparison, for native albumin, we determined an average size of $5.8 \pm 1.5 \mathrm{~nm}$.

3.2. Ga-68 and Cu-64 Labeling of HSA Conjugates. Ferreira et al. reported on the labeling of NOTA with Ga-68 in acetate buffer [16]. A pH of 4-5 and a reaction time of $5 \mathrm{~min}$ (room temperature) were found to be optimal conditions for labeling. According to this published data, we performed Gallium68 labeling in a similar manner using $0.5 \mathrm{M}$ acetate buffer $\mathrm{pH}$ 4,5 , and 6 , respectively. A buffer $\mathrm{pH}$ of 5 revealed the highest labeling yield. After $15 \mathrm{~min}$ at room temperature, the labeling yield after EDTA quenching of the remaining [Ga-68] $\mathrm{Ga}^{3+}$ exceeded $95 \%$ for NOTA-HSA, NOTA-HSA denat $_{\text {, NOTA- }}$ Man-HSA, and NOTA-Man-HSA ${ }_{\text {denat }}$, determined by thin layer chromatography.

To verify the particle size distribution of our labeled HSA conjugates, we filtered an aliquot of the labeling mixture. For these experiments, we used polycarbonate filters with a defined pore size of $80 \mathrm{~nm}$. For all the four conjugates, 97\%$99 \%$ of the radioactivity passed the filter membrane and was detected in the eluate, indicating a particle size smaller than $80 \mathrm{~nm}$. HSA colloids were labeled with Cu-64 under identical 


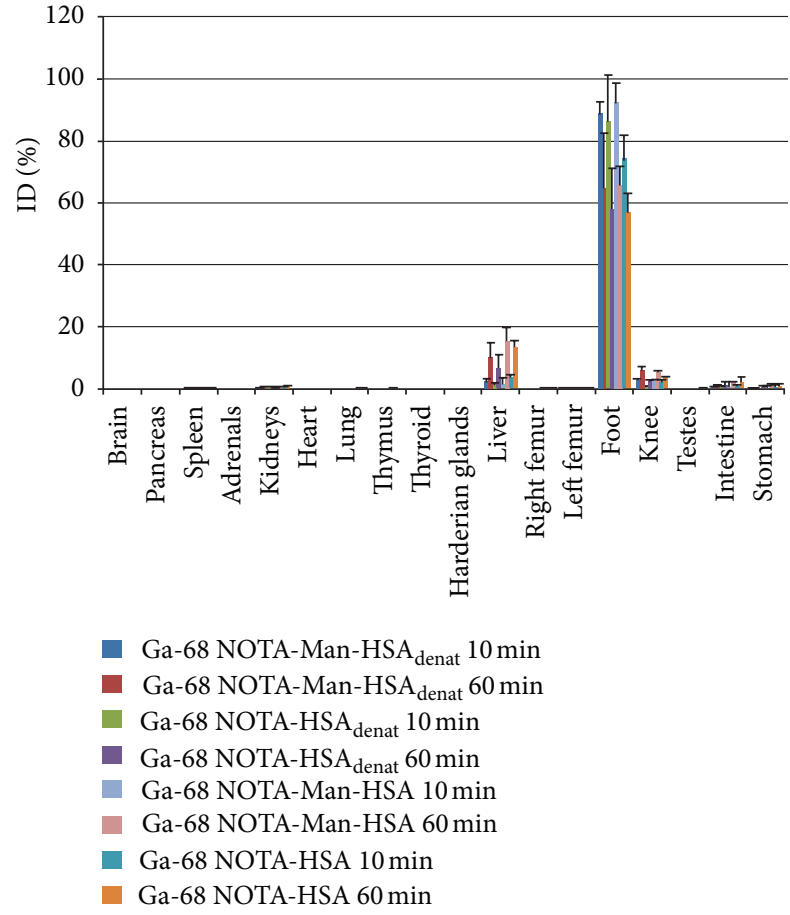

FIGURE 2: Biodistribution of Ga-68-labeled albumin derivatives after subcutaneous injection into the foot pad of Wistar rats $(n=4)$.

conditions with the same high yield. For animal experiments, $1 \mathrm{~mL}$ of HEPES buffer $(1 \mathrm{M}, \mathrm{pH} 8)$ was added to the reaction mixture after labeling, resulting in a $\mathrm{pH}$ of $\mathrm{ca}$. 7. This does not affect the integrity and the purity of the product, as determined by TLC and filtration.

\subsection{Biodistribution Studies of $\mathrm{Ga}-68$ - and Cu-64-Labeled HSA} Conjugates. The rat model was used to analyze the lymphatic transport of the tracers and their uptake in lymph nodes $[17,18]$. Labeled colloids were subcutaneously injected into the foot pad of rats. Colloids are transported to some extent via the lymph vessels. The first passed lymph node (sentinel lymph node) is the popliteal node located behind the knee joint [19]. Analogously to Weiss et al. [18], we separated the whole knee to determine the activity of the popliteal lymph node. Biodistribution was analyzed 10 and $60 \mathrm{~min}$ after injection (Figure 2).

Noticeable activity was found in the foot pad (injection site), knee (popliteal lymph node), and liver. Activity in other organs and tissues was marginal. For all the tested compounds, the activity in the foot pad decreased by ca. $20 \%-25 \%$ ID from 10 to $60 \mathrm{~min}$ p.i., whereas an increase is observed in the popliteal lymph node (knee) and the liver. The uptake in the liver and the transport from the injection site are similar for all conjugates. On the other hand, differences are seen in the lymph node uptake. According to a receptor-mediated mechanism triggered by mannose, conjugates containing mannose are preferably taken up by phagocytosis in comparison to the corresponding conjugate

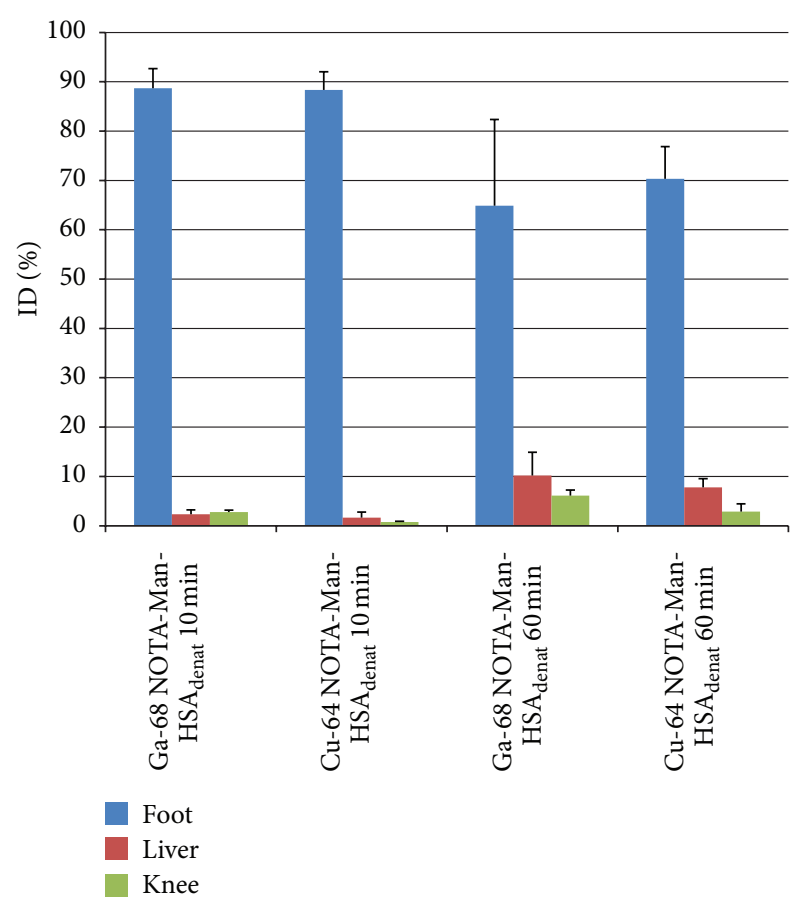

Figure 3: Comparison of Cu-64- and Ga-68-labeled denatured NOTA-Man-HSA after subcutaneous injection into the foot pad of Wistar rats.

(denatured/nondenatured) without mannose units. Furthermore, denaturation by heat treatment seems to have a positive influence on the uptake of the mannosylated conjugate $60 \mathrm{~min}$ p.i. compared to its nondenatured counterpart, although the difference is rather small. Among the four tested conjugates, NOTA-Man-HSA $\mathrm{denat}_{\text {at }}$ revealed the highest lymph node uptake of $2.78 \% \pm 0.38 \%$ ID and $6.13 \% \pm$ $1.13 \%$ ID 10 and $60 \mathrm{~min}$ p.i., respectively. This compound was subsequently labeled with $\mathrm{Cu}-64$. $\mathrm{Cu}-64$ was utilized to further characterize the colloids, taking advantage of better image resolution and longer half-life of $\mathrm{Cu}-64$ in comparison with Ga-68. Essentially, the same biodistribution patterns were found after subcutaneous injection of $\mathrm{Cu}-64$ and Ga-68-labeled NOTA-Man-HSA denat into the foot pad (Figure 3), indicating that the radiolabeling with either of the two radionuclides does not significantly alter the biodistribution. The small differences between the Ga-68- and the $\mathrm{Cu}$-64-labeled agent in Figure 3 are most probably due to different mobility behavior of the rats in both groups. The transport of colloids within the lymph vessels is promoted by muscle contraction. Figure 5 reveals more precise results under equivalent conditions for both groups, because in this experiment animals were anaesthetized throughout the whole experiment (vide infra).

In further experiments, PET data of $\mathrm{Ga}-68$ - and $\mathrm{Cu}$ 64-labeled denatured NOTA-Man-HSA were acquired up to 120 and 240 min p.i., respectively (Figure 4 ). As expected, both tracers show similar distribution patterns, reflecting the results of the biodistribution studies. The main activity is found in the injection site (foot). At later time points, also 
Ga-68 NOTA-Man-HSA denat

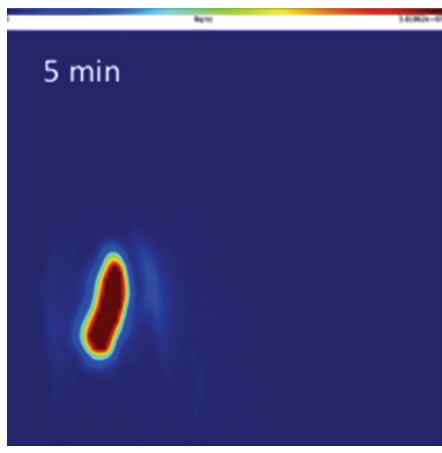

Cu-64 NOTA-Man-HSA denat

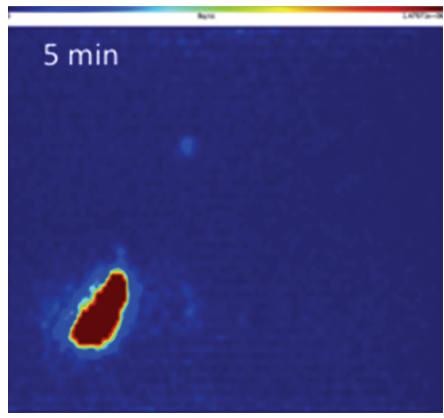

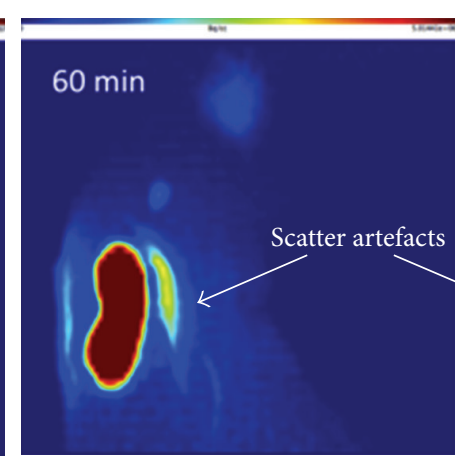
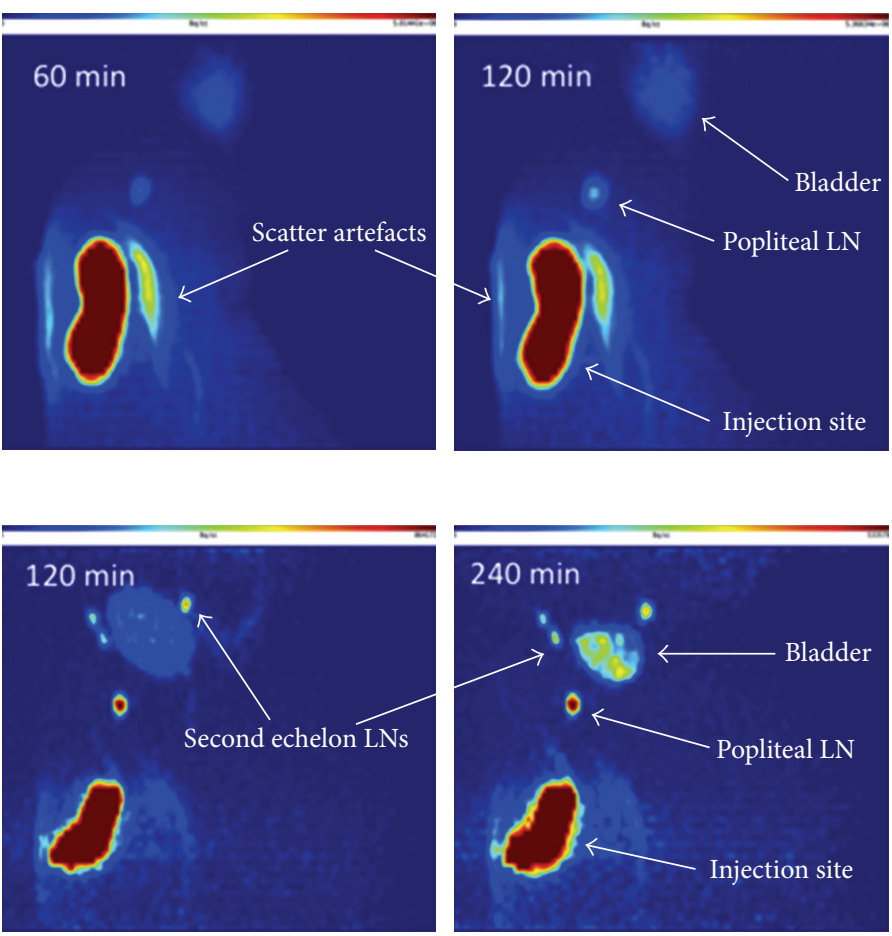

FIGURE 4: Coronal PET images of Ga-68- and Cu-64-labeled denatured NOTA-Man-HSA after subcutaneous injection into the foot pad of rats.

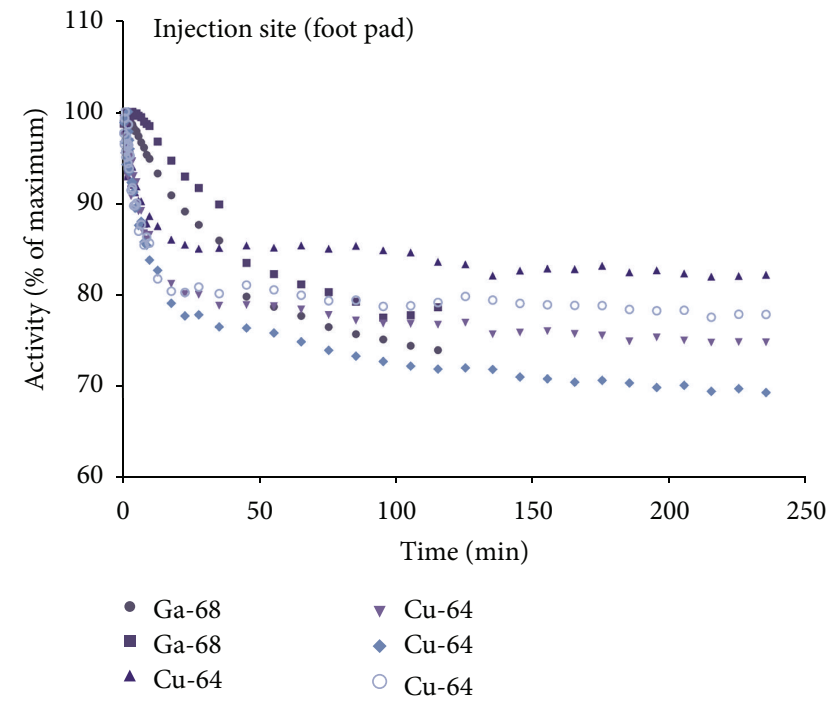

(a)

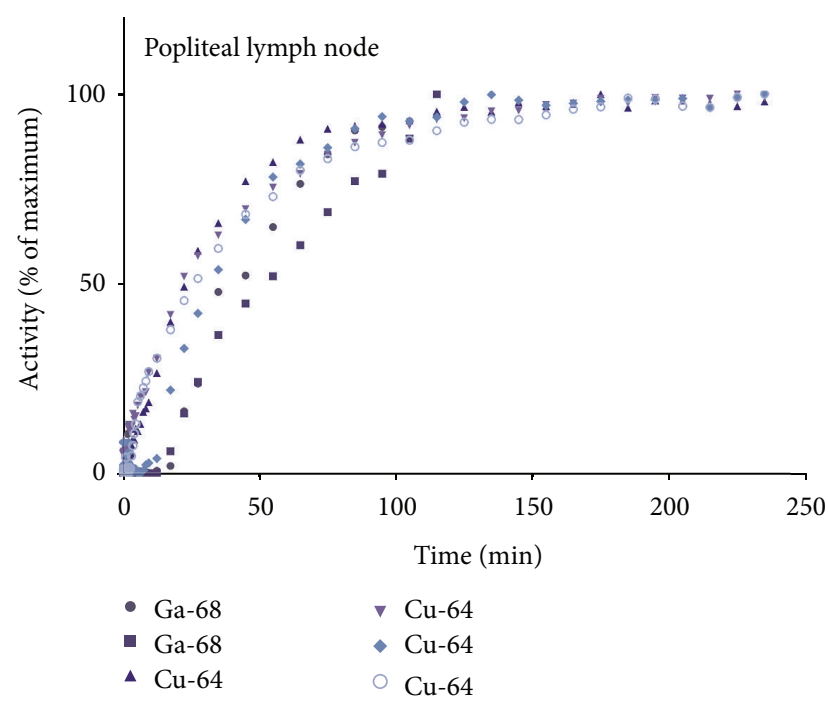

(b)

FIGURE 5: Normalized time-dependent radioactivity uptake in the foot pad and the popliteal lymph node after subcutaneous injection of Ga-68- $(n=2)$ and Cu-64- $(n=4)$ labeled denatured NOTA-Man-HSA into the foot pad of rats.

the bladder becomes slightly visible. The popliteal lymph node is visible 60 and $120 \mathrm{~min}$ p.i. for the Ga-68-labeled compound and $120 \mathrm{~min}$ and $240 \mathrm{~min}$ p.i. in the case of $\mathrm{Cu}-$ 64. The normalized activity curves show a slow radioactivity accumulation within the lymph node. The maximum activity plateau is reached after approximately $120 \mathrm{~min}$ in all tested individuals, independent of the radiolabel (Figure 5).
Copper-64 images are characterized by higher resolution and lower scatter artefacts due to the lower $\beta^{+}$-energy in comparison to Ga-68. This might also be the reason why in the case of $\mathrm{Cu}-64$ second echelon lymph nodes become visible $120 \mathrm{~min}$ p.i.

Lymph node uptake of Ga-68 NOTA-Man-HSA after subcutaneous injection is also presented by Choi et al. [12]. Their 


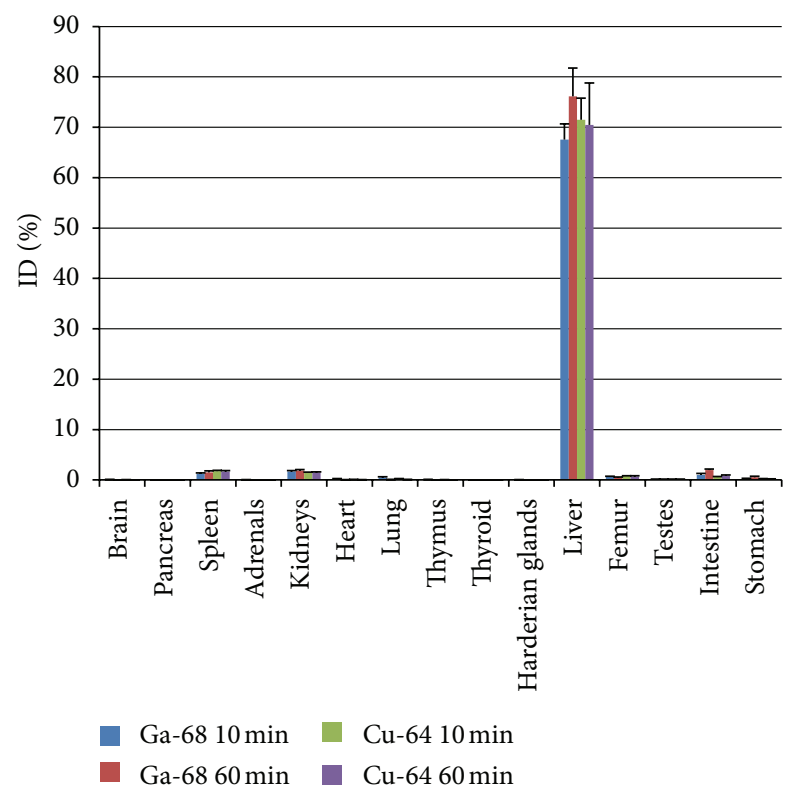

Figure 6: Biodistribution of Cu-64- and Ga-68-labeled denatured NOTA-Man-HSA after intravenous injection into the tail vein of Wistar rats.

data are obtained from dynamic micro-PET imaging up to 10 min p.i. A more or less constant popliteal uptake of approx. $5 \%$ ID is observed as soon as $1 \mathrm{~min}$ p.i., which is unusual for a biological transport mechanism and is not in accordance to our finding of slow increase of activity accumulation within the lymph node (Figure 5). Data on the uptake up to several hours p.i. are important for intraoperative detection of lymph nodes by hand-held beta probes. In contrast to Tc-99m-labeled colloids where lymph node dissection is usually performed $15-24 \mathrm{~h}$ p.i. $[5,20]$, intraoperative node detection has to be performed immediately after imaging in the case of gallium-68 due to its shorter physical half-life. We demonstrated high and persistent lymph node uptake of denatured NOTA-Man-HSA up to 240 min p.i.

Fast transport from the injection site is another crucial factor. For example, in a study of Vermeeren et al. in 6 out of 46 patients, sentinel lymph nodes could not be detected by planar imaging due to their close proximity to the injection area [2]. In our studies, the activity of denatured NOTA-ManHSA at the site of injection decreased from $88.7 \% \pm 4.5 \%$ ID $10 \mathrm{~min}$ p.i. to $64.9 \% \pm 17.5 \%$ ID. This is significantly lower than that of the currently used Tc-99m-labeled colloid, where almost the entire injected dose remains at the injection site [20].

Gallium-68- and copper-64-labeled NOTA-Man$\mathrm{HSA}_{\text {denat }}$ were also characterized by their biodistribution after intravenous injection 10 and $60 \mathrm{~min}$ p.i. (Figure 6). As expected, both tracers show similar distribution. The main activity was found in the liver indicating an uptake by the reticuloendothelial system. The uptake in spleen, kidneys, and intestine is rather low (ca. 1\% ID). Other organs are negligible.

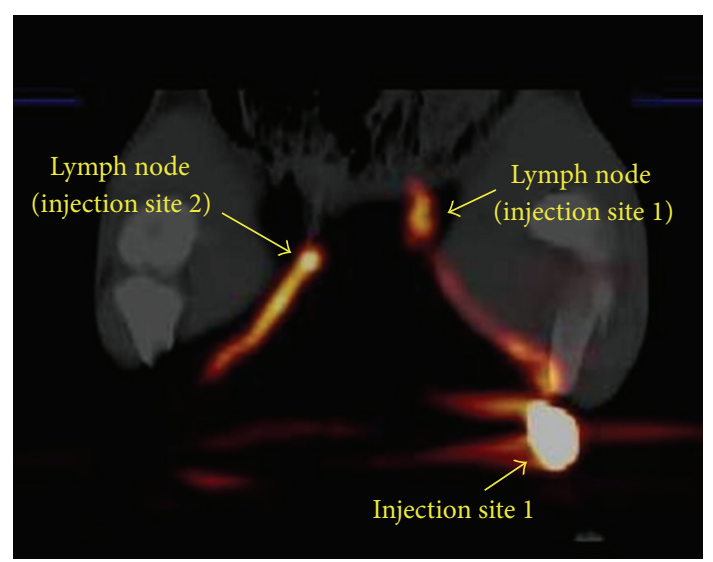

FIgURE 7: Transversal PET/CT section image 15 min after injection of $45 \mathrm{MBq}$ Ga-68 NOTA-HSA denat into each hind leg of a pig (injection site 2 is located outside the section and therefore is not visible).

Furthermore, a pig model was utilized to create PET/CT images of the lymphatic transport and lymph node uptake. The conjugate with the highest uptake in the rat popliteal lymph node, Ga-68-labeled NOTA-Man-HSA denat, was injected in both extremities according to the procedure of Tafra et al. [15]. Clearly, the lymphatic transport and the uptake in the sentinel lymph nodes could be visualized on early images 15 min p.i. (Figure 7).

The mean uptake of both injections into the sentinel node was $1.2 \%$ after $15 \mathrm{~min}$ and $2.0 \%$ after $60 \mathrm{~min}$. In another published study in which a pig model was used, lymph node uptakes of $1.32 \%-2.04 \%$ ID were found for Lymphoseek and $0.63 \%-2.35 \%$ ID for filtered Tc-99m sulphur colloid [21]. In comparison, average lymph node uptakes of $1 \%$ ID for HSA nanocolloids [20], 0.65\% ID [22] and $0.85 \%$ ID for sulphur colloids [23], and 0.73\% ID for Lymphoseek [23] are reported for patients with malignant melanoma, mammalian carcinoma, or prostate cancer. These similar values can be explained by saturation of the limited number of phagocytic cells (macrophages or dendritic cells) within the lymph node [24].

\section{Conclusion}

Four different conjugates based on NOTA-human serum albumin have been synthesized and labeled with Ga-68 and $\mathrm{Cu}-64$, respectively. The effects of mannosyl units and denaturation were studied after subcutaneous injection into the foot pad of rats. Distinct uptake in the popliteal lymph node was observed for all tested compounds. Mannosylation could be identified to positively influence the uptake in the lymph node. The effect of denaturation by heat treatment in the case of mannosylated compounds is rather low. However, heattreated HSA colloids bearing mannosyl units (NOTA-Man$\mathrm{HSA}_{\text {denat }}$ ) revealed the highest lymph node uptake of $6.13 \% \pm$ $1.13 \%$ ID 60 min p.i. After the intravenous injection in 
rats, the majority of Ga-68- and Cu-64-labeled NOTA-Man$\mathrm{HSA}_{\text {denat }}$ were taken up quickly by the reticuloendothelial system of the liver.

PET experiments in rats revealed similar distribution pattern for Ga-68- and Cu-64-labeled NOTA-Man-HSA denat The first lymph node in the drainage basin reaches its maximum activity after approx. 120 min and remained constant for the copper-64 compound at least up to $240 \mathrm{~min}$ p.i.

The feasibility of lymphatic mapping with Ga-68-labeled NOTA-Man-HSA denat was furthermore demonstrated by PET/CT imaging using a pig model, where $2 \%$ of the injected dose was found in the sentinel lymph node $60 \mathrm{~min}$ p.i.

\section{Acknowledgments}

This work was financially supported by the European Regional Development Fund (ERDF) and the Free State of Saxony (Project no. 12629/2089). The authors wish to thank Regina Herrlich for the assistance in the radiopharmacological investigations.

\section{References}

[1] I. M. C. Van Der Ploeg, O. E. Nieweg, B. B. R. Kroon et al., "The yield of SPECT/CT for anatomical lymphatic mapping in patients with breast cancer," European Journal of Nuclear Medicine and Molecular Imaging, vol. 36, no. 6, pp. 903-909, 2009.

[2] L. Vermeeren, R. A. Valdés Olmos, W. Meinhardt et al., "Value of SPECT/CT for detection and anatomic localization of sentinel lymph nodes before laparoscopic sentinel node lymphadenectomy in prostate carcinoma," Journal of Nuclear Medicine, vol. 50, no. 6, pp. 865-870, 2009.

[3] M. Weiss, R. A. Schmid, C. Kunte, B. Konz, and K. Hahn, "First experiences with a new radiopharmaceutical for sentinel lymph node detection in malignant melanoma: ${ }^{99 m} \mathrm{Tc}$ colloidal rhenium sulphide," NuklearMedizin, vol. 43, no. 1, pp. 10-15, 2004.

[4] A. E. Hawley, S. S. Davis, and L. Illum, "Targeting of colloids to lymph nodes: influence of lymphatic physiology and colloidal characteristics," Advanced Drug Delivery Reviews, vol. 17, no. 1, pp. 129-148, 1995.

[5] G. Mariani, L. Moresco, G. Viale et al., "Radioguided sentinel lymph node biopsy in breast cancer surgery," Journal of Nuclear Medicine, vol. 42, no. 8, pp. 1198-1215, 2001.

[6] H. Vogt, M. Schmidt, R. Bares et al., "Verfahrensanweisung für die nuklearmedizinische wächter-lymphknoten-diagnostik," Nuklearmedizin, vol. 49, pp. 167-172, 2010.

[7] V. K. Sondak, S. Marzban, C. J. Rich, J. L. Messina, and J. S. Zager, "Identification of melanoma sentinel nodes with lymphoseek: phase III clinical trial results at moffitt cancer center," Annals of Surgical Oncology, vol. 17, p. S111, 2010.

[8] K. K. Limmer, S. P. Povoski, H. Krontiras et al., "Phase III results comparing lymphoseek with blue dye in detection of the sentinel lymph node in breast cancer," Cancer Research, vol. 69, 3, no. 24, p. 514S, 2009.

[9] A. M. Wallace, C. K. Hoh, D. R. Vera, D. D. Darrah, and G. Schulteis, "Lymphoseek: a molecular radiopharmaceutical for sentinel node detection," Annals of Surgical Oncology, vol. 10, no. 5, pp. 531-538, 2003.
[10] U. Ganswindt, D. Schilling, A. C. Müller, R. Bares, P. Bartenstein, and C. Belka, "Distribution of prostate sentinel nodes: a SPECT-derived anatomic atlas," International Journal of Radiation Oncology Biology Physics, vol. 79, no. 5, pp. 1364-1372, 2011.

[11] D. A. Heuveling, G. M. W. Visser, M. Baclayon et al., " ${ }^{89} \mathrm{Zr}$ nanocolloidal albumin-based PET/CT lymphoscintigraphy for sentinel node detection in head and neck cancer: preclinical results," Journal of Nuclear Medicine, vol. 52, no. 10, pp. 15801584, 2011.

[12] J. Y. Choi, J. M. Jeong, B. C. Yoo et al., "Development of ${ }^{68} \mathrm{Ga}$ labeled mannosylated human serum albumin (MSA) as a lymph node imaging agent for positron emission tomography," Nuclear Medicine and Biology, vol. 38, no. 3, pp. 371-379, 2011.

[13] M. Kühne, Z. Györgydeak, and T. K. Lindhorst, "A simple method for the preparation of glycosyl isothiocyanates," Synthesis, no. 6, pp. 949-951, 2006.

[14] S. Thieme, M. Walther, H.-J. Pietzsch et al., "Module-assisted preparation of ${ }^{64} \mathrm{Cu}$ with high specific activity," Applied Radiation and Isotopes, vol. 70, no. 4, pp. 602-608, 2012.

[15] L. Tafra, A. N. Chua, P. C. Ng, D. Aycock, M. Swanson, and D. Lannin, "Filtered versus unfiltered technetium sulfur colloid in lymphatic mapping: a significant variable in a pig model," Annals of Surgical Oncology, vol. 6, no. 1, pp. 83-87, 1999.

[16] C. L. Ferreira, E. Lamsa, M. Woods et al., "Evaluation of bifunctional chelates for the development of gallium-based radiopharmaceuticals," Bioconjugate Chemistry, vol. 21, no. 3, pp. 531-536, 2010.

[17] E. G. F. Núñez, B. L. Faintuch, R. Teodoro et al., "Influence of colloid particle profile on sentinel lymph node uptake," Nuclear Medicine and Biology, vol. 36, no. 7, pp. 741-747, 2009.

[18] M. Weiss, F. J. Gildehaus, K. Brinkbäumer, M. Makowski, and K. Hahn, "Lymph kinetics with technetium-99m labeled radiopharmaceuticals: animal studies," NuklearMedizin, vol. 44, no. 4, pp. 156-165, 2005.

[19] N. L. Tilney, "Patterns of lymphatic drainage in the adult laboratory rat," Journal of Anatomy, vol. 109, no. 3, pp. 369-383, 1971.

[20] W. A. Waddington, M. R. S. Keshtgar, I. Taylor, S. R. Lakhani, M. D. Short, and P. J. Ell, "Radiation safety of the sentinel lymph node technique in breast cancer," European Journal of Nuclear Medicine, vol. 27, no. 4, pp. 377-391, 2000.

[21] S. J. Ellner, J. Méndez, D. R. Vera, C. K. Hoh, W. L. Ashburn, and A. M. Wallace, "Sentinel lymph node mapping of the colon and stomach using lymphoseek in a pig model," Annals of Surgical Oncology, vol. 11, no. 7, pp. 674-681, 2004.

[22] T. de Paulis, "Drug evaluation: lymphoseek-neoprobe's sentinel lymph node imaging agent for use in cancer patients," Current Opinion in Investigational Drugs, vol. 7, no. 12, pp. 11001107, 2006.

[23] A. M. Wallace, C. K. Hoh, S. J. Ellner, D. D. Darrah, G. Schulteis, and D. R. Vera, "Lymphoseek: a molecular imaging agent for melanoma sentinel lymph node mapping," Annals of Surgical Oncology, vol. 14, no. 2, pp. 913-921, 2007.

[24] H. Schöder, E. C. Glass, A. P. Pecking et al., "Molecular targeting of the lymphovascular system for imaging and therapy," Cancer and Metastasis Reviews, vol. 25, no. 2, pp. 185-201, 2006. 

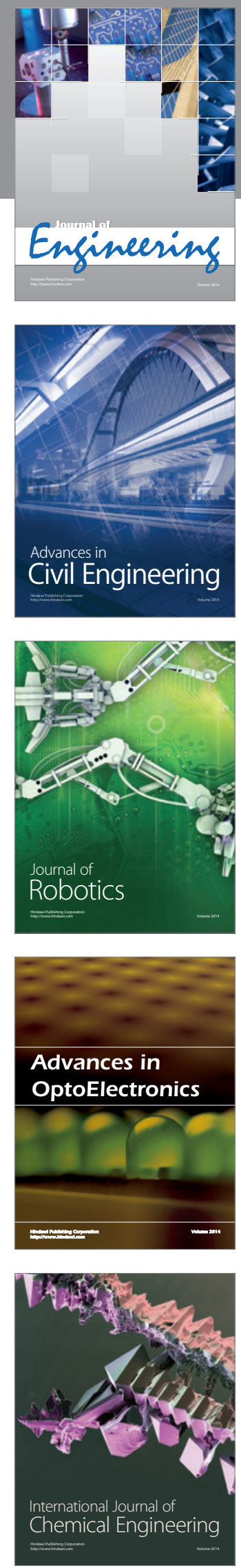

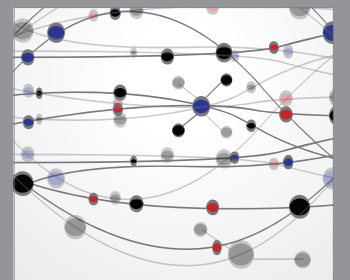

The Scientific World Journal
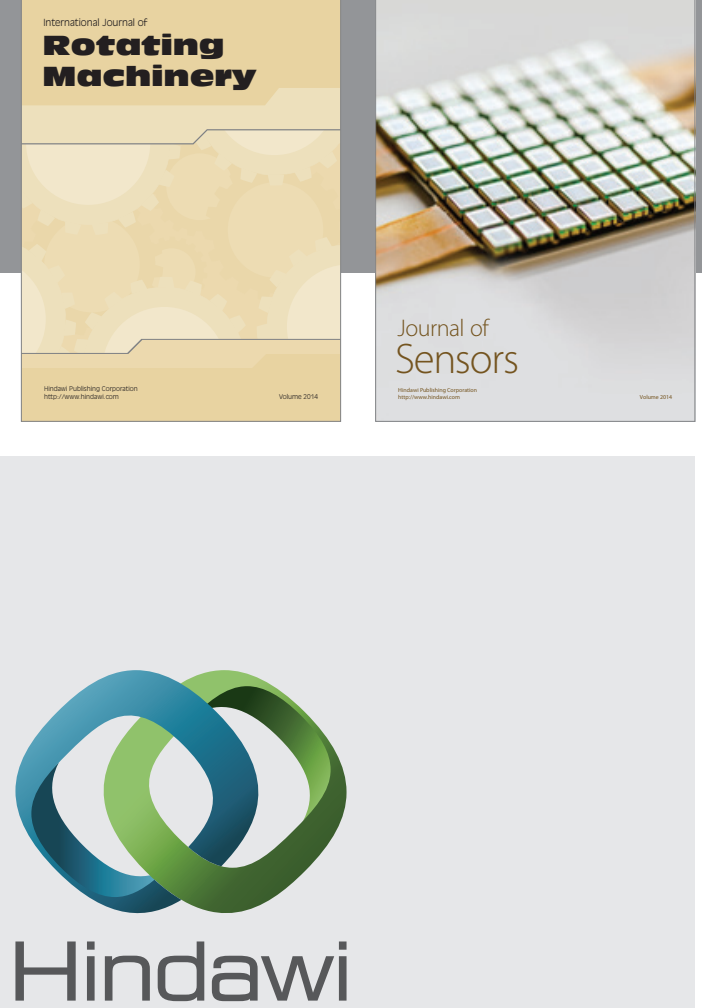

Submit your manuscripts at http://www.hindawi.com
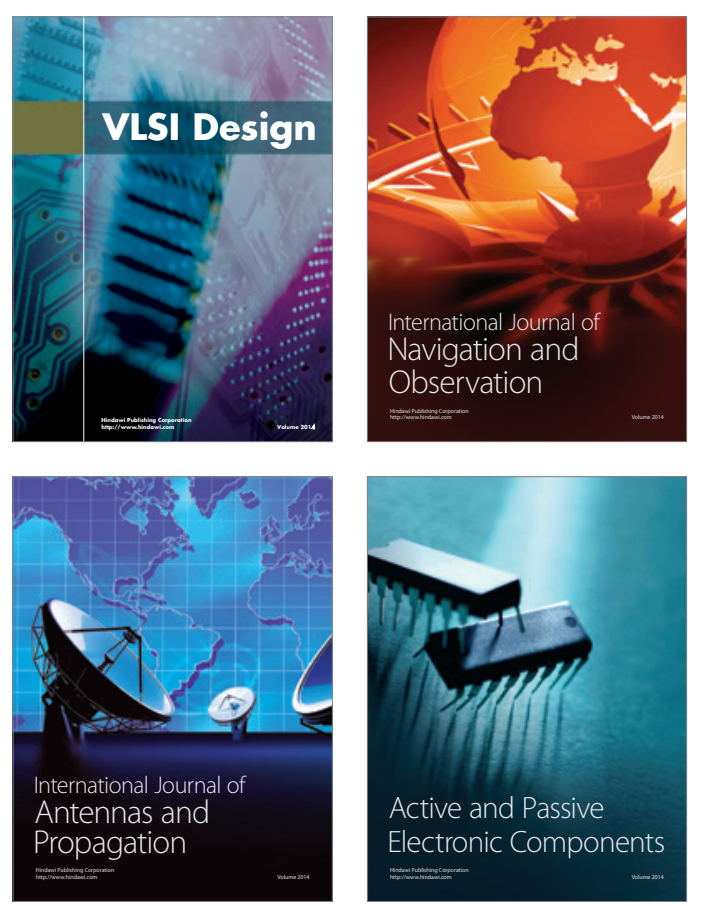
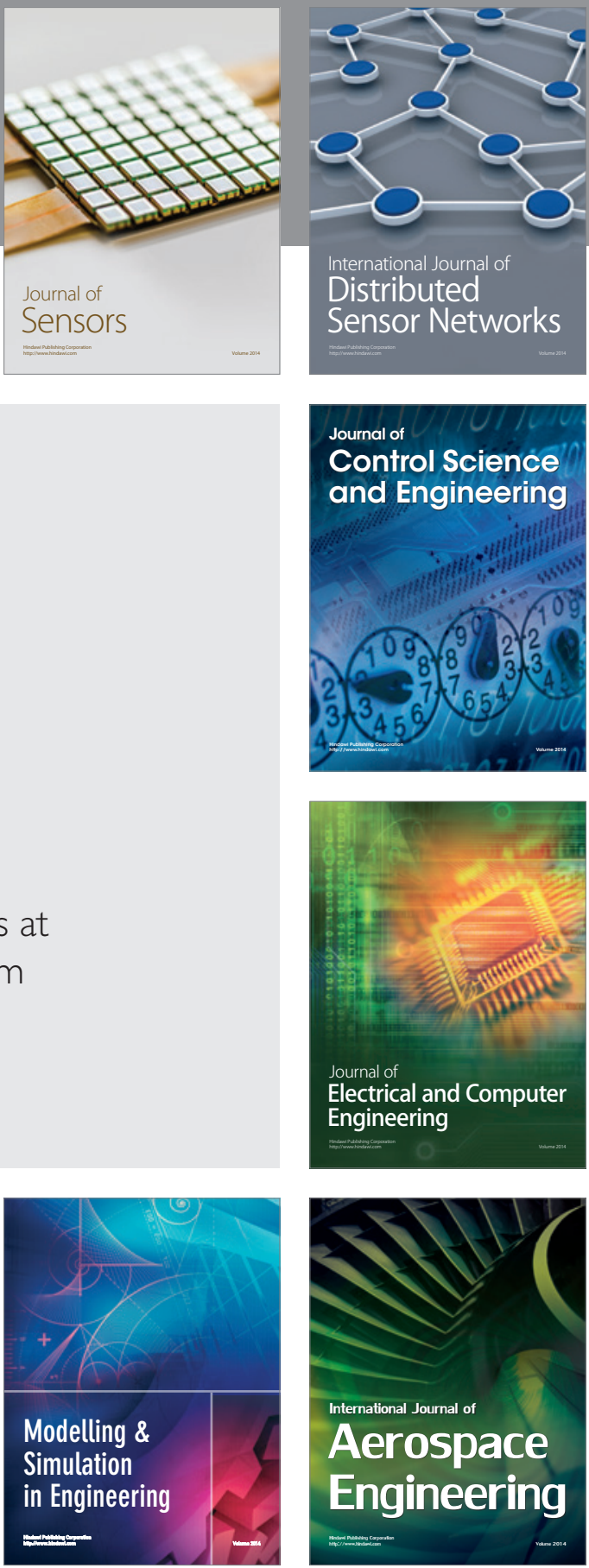

Journal of

Control Science

and Engineering
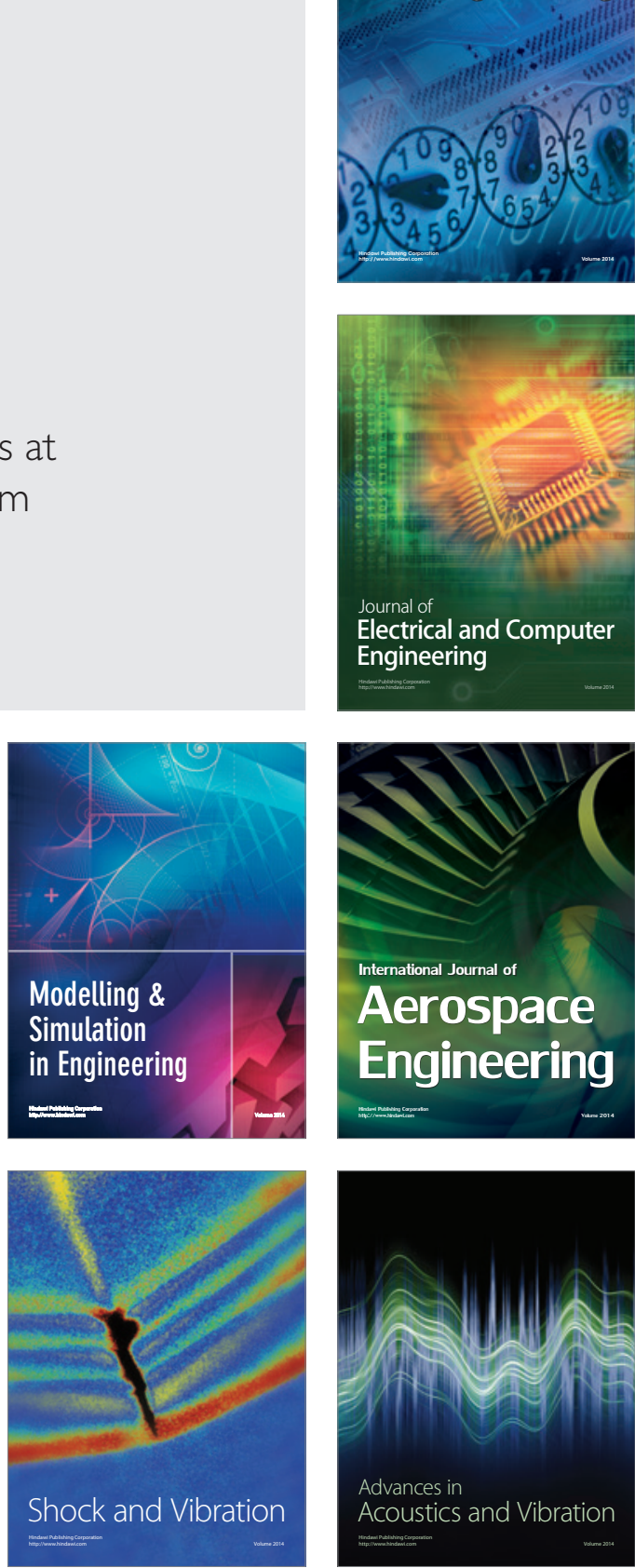